\title{
A Note on Specializations of Grothendieck Polynomials
}

\author{
Neil J.Y. Fan ${ }^{1}$ and Peter L. Guo ${ }^{2}$ \\ ${ }^{1}$ Department of Mathematics \\ Sichuan University, Chengdu, Sichuan 610064, P.R. China \\ ${ }^{2}$ Center for Combinatorics, LPMC \\ Nankai University, Tianjin 300071, P.R. China \\ ${ }^{1}$ fan@scu.edu.cn, ${ }^{2}$ lguo@nankai.edu.cn
}

\begin{abstract}
Buch and Rimányi proved a formula for a specialization of double Grothendieck polynomials based on the Yang-Baxter equation related to the degenerate Hecke algebra. A geometric proof was found by Yong and Woo by constructing a Gröbner basis for the Kazhdan-Lusztig ideals. In this note, we give an elementary proof for this formula by using only divided difference operators.
\end{abstract}

\section{Introduction}

Let $S_{n}$ denote the symmetric group of permutations of $\{1,2, \ldots, n\}$. For a permutation $w \in S_{n}$, the double Grothendieck polynomial $\mathfrak{G}_{w}(x ; y)$ introduced by Lascoux and Schützenberger [12] is the polynomial representative of the class of the Schubert variety for $w$ in the equivariant $K$-theory of the flag manifold. Write a permutation $v \in S_{n}$ in one-line notation, that is, write $v=v(1) v(2) \cdots v(n)$. The specialization

$$
\mathfrak{G}_{w}\left(y_{v} ; y\right):=\mathfrak{G}_{w}\left(y_{v(1)}, \ldots, y_{v(n)} ; y\right)
$$

of $\mathfrak{G}_{w}(x ; y)$ obtained by replacing $x_{i}$ with $y_{v(i)}$ gives the restriction of this class to the fixed point corresponding to $v$. Buch and Rimányi [4] proved a formula for $\mathfrak{G}_{w}\left(y_{v} ; y\right)$ based on the Yang-Baxter equation related to the degenerate Hecke algebra. Buch and Rimányi [4] also pointed out various important applications of this formula. By constructing a Gröbner basis for the Kazhdan-Lusztig ideals, Yong and Woo [15] found a geometric explanation for the Buch-Rimányi formula.

In this note, we give an elementary proof of the Buch-Rimányi formula by using only divided difference operators. As observed by Buch and Rimányi [4, Corollary 2.3], the classical pipe dream (or, RC-graph) formula of $\mathfrak{G}_{w}(x ; y)$ (see for example [10, Corollary 5.4], [13, Theorem 6.3]) can be directly obtained from the specialization $\mathfrak{G}_{w}\left(y_{v} ; y\right)$. Hence our approach implies that the pipe dream formula for double Grothendieck polynomials can be derived directly from divided difference operators. 


\section{The Buch-Rimányi formula}

Fix a nonnegative integer $n$. For $1 \leq i<j \leq n$, let $t_{i j}$ denote the transposition $(i, j)$ in $S_{n}$. So, if $w \in S_{n}$, then $w t_{i j}$ is the permutation obtained from $w$ by interchanging $w(i)$ and $w(j)$, while $t_{i j} w$ is obtained from $w$ by interchanging the values $i$ and $j$. For example, for $w=2143$, we have $w t_{13}=4123$ and $t_{13} w=2341$. Write $s_{i}$ for the adjacent transposition $(i, i+1)$. Each permutation can be written as a product of adjacent transpositions. The length $\ell(w)$ of a permutation $w$ is the minimum $k$ such that $w=s_{i_{1}} s_{i_{2}} \cdots s_{i_{k}}$, and in this case, $\left(s_{i_{1}}, s_{i_{2}}, \ldots, s_{i_{k}}\right)$ is called a reduced word of $w$. It is well known that the length $\ell(w)$ is equal to the number of pairs $(i, j)$ such that $i<j$ and $w(i)>w(j)$ :

$$
\ell(w)=\#\{(i, j): 1 \leq i<j \leq n, w(i)>w(j)\} .
$$

Hence, it is clear that $\ell\left(w s_{i}\right)=\ell(w)+1$ if and only if $w(i)<w(i+1)$, while $\ell\left(w s_{i}\right)=\ell(w)-1$ if and only if $w(i)>w(i+1)$.

Let $\mathbb{Z}\left[x^{ \pm}, y^{ \pm}\right]$denote the ring of Laurent polynomials in the $2 n$ commuting indeterminates $x_{1}, \ldots, x_{n}, y_{1}, \ldots, y_{n}$. For a Laurent polynomial $f(x, y) \in \mathbb{Z}\left[x^{ \pm}, y^{ \pm}\right]$, the divided difference operator $\partial_{i}$ acting on $f(x, y)$ is defined by

$$
\partial_{i} f=\left(f-s_{i} f\right) /\left(x_{i}-x_{i+1}\right),
$$

where $s_{i} f$ is obtained from $f$ by interchanging $x_{i}$ and $x_{i+1}$. It is easy to check that $\partial_{i} f$ is still a Laurent polynomial. Let $w_{0}=n \cdots 21$ be the longest permutation in $S_{n}$. Set

$$
\mathfrak{G}_{w_{0}}(x ; y)=\prod_{i+j \leq n}\left(1-\frac{y_{j}}{x_{i}}\right) \text {. }
$$

For $w \neq w_{0}$, choose an adjacent transposition $s_{i}$ such that $\ell\left(w s_{i}\right)=\ell(w)+1$. Let $\pi_{i}=\partial_{i} x_{i}$ and define

$$
\mathfrak{G}_{w}(x ; y)=\pi_{i} \mathfrak{G}_{w s_{i}}(x ; y)=\frac{x_{i} \mathfrak{G}_{w s_{i}}(x ; y)-x_{i+1} \mathfrak{G}_{w s_{i}}\left(\ldots, x_{i+1}, x_{i}, \ldots ; y\right)}{x_{i}-x_{i+1}} .
$$

The above definition is independent of the choice of $s_{i}$ since the operators $\pi_{i}$ satisfy the Coxeter relations: $\pi_{i} \pi_{j}=\pi_{j} \pi_{i}$ for $|i-j|>1$, and $\pi_{i} \pi_{i+1} \pi_{i}=\pi_{i+1} \pi_{i} \pi_{i+1}$, see for example [14, (2.14)].

We remark that there are other equivalent definitions for double Grothendieck polynomials. The definition adopted here implies that $\mathfrak{G}_{w}(x ; y)$ are Laurent polynomials. The double Grothendieck polynomials $\mathfrak{L}_{w}^{(-1)}(y ; x)$ defined in [5] are legitimate polynomials, which can be obtained from $\mathfrak{G}_{w}(x ; y)$ by replacing $x_{i}$ and $y_{i}$ respectively with $\frac{1}{1-x_{i}}$ and $1-y_{i}$. It should also be noticed that $\mathfrak{G}_{w}\left(x^{-1} ; y^{-1}\right)$ are the double Grothendieck polynomials used in [9], and $\mathfrak{G}_{w}\left(x^{-1} ; y\right)$ are the double Grothendieck polynomials appearing in [10]. It is worth mentioning that the double Schubert polynomial $\mathfrak{S}_{w}(x ; y)$ is the lowest degree homogeneous component of $\mathfrak{L}_{w}^{(-1)}(y ; x)$, see [1,2, 6, 7, 11] for combinatorial constructions of Schubert polynomials.

To describe the Buch-Rimányi formula, consider the left-justified array $\Delta_{n}$ with $n-i$ squares in row $i$. Let $w=w(1) w(2) \cdots w(n) \in S_{n}$. For $1 \leq i \leq n$, let

$$
I(w, i)=\{w(j): j>i, w(j)<w(i)\}
$$


be the set of entries in $w$ that are smaller than $w(i)$ but appear to the right of $w(i)$. Set $c(w, i)=|I(w, i)|$. It is clear that $0 \leq c(w, i) \leq n-i$. Let $D(w)$ be the subset of $\Delta_{n}$ consisting of the first $c(w, i)$ squares in the $i$-th row of $\Delta_{n}$, where $1 \leq i \leq n$. Note that $D(w)$ corresponds to the bottom RC-graph of $w$, as defined by Bergeron and Billey [1]. Assume that the values in $I(w, i)$ are

$$
w\left(j_{1}\right)<w\left(j_{2}\right)<\cdots<w\left(j_{c(w, i)}\right) .
$$

For a square $B \in D(w)$ in row $i$ and column $k$, equip $B$ with the weight

$$
\mathrm{wt}(B)=1-\frac{y_{w\left(j_{k}\right)}}{y_{w(i)}}
$$

see Figure 2.1 for an illustration.

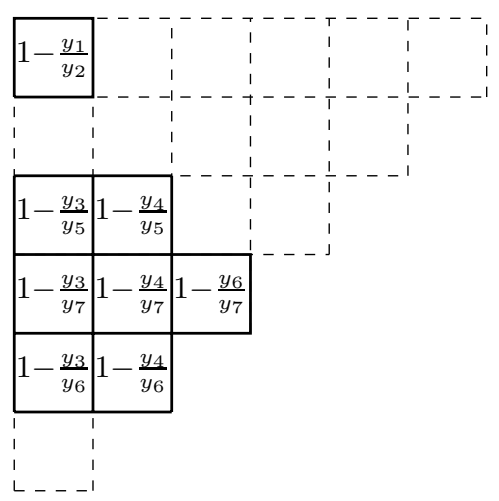

Figure 2.1: Weights of squares of $D(w)$ for $w=2157634$.

Given a subset $D$ of $D(w)$, one can generate a word, denoted word $(D)$, as follows. Label the square of $D(w)$ in row $i$ and column $k$ by the simple transposition $s_{i+k-1}$, see Figure 2.2 for an illustration. Then $\operatorname{word}(D)$ is obtained by reading off the labels of the squares in $D$

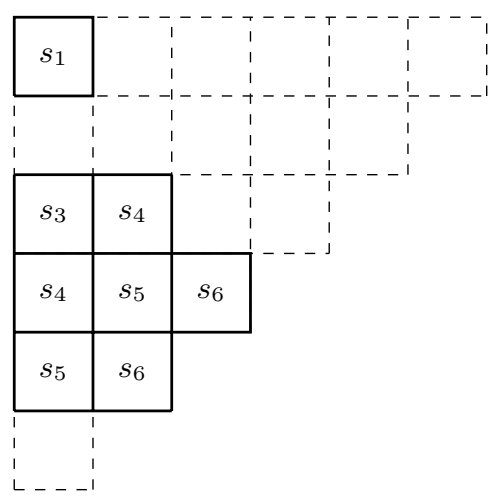

Figure 2.2: Labels of the squares of $D(w)$ for $w=2157634$.

along the rows from top to bottom and right to left. For example, for the diagram $D=D(w)$ in Figure 2.2, we have

$$
\operatorname{word}(D)=\left(s_{1}, s_{4}, s_{3}, s_{6}, s_{5}, s_{4}, s_{6}, s_{5}\right)
$$


A word $\left(s_{i_{1}}, s_{i_{2}}, \ldots, s_{i_{m}}\right)$ is called a Hecke word of a permutation $u$ of length $m$ if

$$
\left(\left(\left(s_{i_{1}} * s_{i_{2}}\right) * s_{i_{3}}\right) * \cdots\right) * s_{i_{m}}=u
$$

where, for a permutation $w$, we define $w * s_{i}$ to be $w$ if $\ell\left(w s_{i}\right)<\ell(w)$ and $w s_{i}$ otherwise. For example, $\left(s_{1}, s_{2}, s_{1}, s_{2}\right)$ is a Hecke word of $u=321$ of length 4 since

$$
\left(\left(s_{1} * s_{2}\right) * s_{1}\right) * s_{2}=\left(\left(s_{1} s_{2}\right) * s_{1}\right) * s_{2}=\left(s_{1} s_{2} s_{1}\right) * s_{2}=s_{1} s_{2} s_{1}=321 .
$$

We note in passing that the operation $*$ can be extended to an associative operation on the whole $S_{n}$; this latter operation is the multiplication in the Hecke algebra associated to $S_{n}$ at $q=0$, see [8, Chapter 7.4]. Hence $*$ satisfies the associative property. This means that the set of permutations in $S_{n}$ forms a monoid structure (0-Hecke monoid) under the operation $*$.

Write Hecke $(D)=u$ if $\operatorname{word}(D)$ is a Hecke word of a permutation $u$. Notice that a Hecke word of $u$ of length $\ell(u)$ is a reduced word of $u$. Note that for any $w \in S_{n}$, the word $\operatorname{word}(D(w))$ is a reduced word of $w$, and therefore, if we multiply the letters of word $(D(w))$ using either the * product or the usual product of $S_{n}$, then we get $w$. That is, $\operatorname{Hecke}(D(w))=w$.

For any $u, v \in S_{n}$, let

$$
\mathcal{H}(u, v)=\{D \subseteq D(v) \mid \operatorname{Hecke}(D)=u\} .
$$

For a subset $D$ of $D(v)$, let

$$
\mathrm{wt}(D)=\prod_{B \in D} \mathrm{wt}(B)
$$

Theorem 2.1 (Buch-Rimányi [4, Theorem 2.1]). For permutations $u, v \in S_{n}$, we have

$$
\mathfrak{G}_{u}\left(y_{v} ; y\right)=\sum_{D \in \mathcal{H}(u, v)}(-1)^{|D|-\ell(u)} \mathrm{wt}(D),
$$

where empty sums are interpreted as 0 .

We remark that in [4], formula (2.4) is described in terms of the notation $C\left(\mathfrak{D}_{v}\right)$ and FKgraphs for $u$ with respect to $\mathfrak{D}_{v}$. With the notation in this note, $D(v)$ can be obtained from $C\left(\mathfrak{D}_{v}\right)$ by first reflecting along the main diagonal and then left-justifying the crossing positions. This operation also establishes a weight preserving bijection between the set $\mathcal{H}(u, v)$ and the set of FK-graphs for $u$ with respect to $\mathfrak{D}_{v}$.

\section{$3 \quad$ Elementary proof of Theorem 2.1}

We need several lemmas which follow directly from the definition of $\mathfrak{G}_{w}(x ; y)$.

Lemma 3.1. Let $v=v^{\prime} s_{i}$ and $\ell(v)>\ell\left(v^{\prime}\right)$. If $\ell\left(u s_{i}\right)<\ell(u)$, then

$$
\mathfrak{G}_{u}\left(y_{v} ; y\right)=\frac{y_{v^{\prime}(i)}}{y_{v^{\prime}(i+1)}} \mathfrak{G}_{u}\left(y_{v^{\prime}} ; y\right)+\left(1-\frac{y_{v^{\prime}(i)}}{y_{v^{\prime}(i+1)}}\right) \mathfrak{G}_{u s_{i}}\left(y_{v^{\prime}} ; y\right)
$$


Proof. Applying (2.2) to $w=u s_{i}$ and substituting $x_{j}$ with $y_{v^{\prime}(j)}$, we have

$$
\mathfrak{G}_{u s_{i}}\left(y_{v^{\prime}} ; y\right)=\frac{y_{v^{\prime}(i)} \mathfrak{G}_{u}\left(y_{v^{\prime}} ; y\right)-y_{v^{\prime}(i+1)} \mathfrak{G}_{u}\left(y_{v} ; y\right)}{y_{v^{\prime}(i)}-y_{v^{\prime}(i+1)}},
$$

which is equivalent to (3.1).

Lemma 3.2. Let $v=v^{\prime} s_{i}$. If $\ell\left(u s_{i}\right)>\ell(u)$, then

$$
\mathfrak{G}_{u}\left(y_{v} ; y\right)=\mathfrak{G}_{u}\left(y_{v^{\prime}} ; y\right)
$$

Proof. Applying (2.2) to $w=u$ and substituting $x_{j}$ with $y_{v(j)}$ and $y_{v^{\prime}(j)}$ respectively, we see that

$$
\begin{aligned}
\mathfrak{G}_{u}\left(y_{v} ; y\right) & =\frac{y_{v(i)} \mathfrak{G}_{u s_{i}}\left(y_{v} ; y\right)-y_{v(i+1)} \mathfrak{G}_{u s_{i}}\left(y_{v^{\prime}} ; y\right)}{y_{v(i)}-y_{v(i+1)}} \\
\mathfrak{G}_{u}\left(y_{v^{\prime}} ; y\right) & =\frac{y_{v^{\prime}(i)} \mathfrak{G}_{u s_{i}}\left(y_{v^{\prime}} ; y\right)-y_{v^{\prime}(i+1)} \mathfrak{G}_{u s_{i}}\left(y_{v} ; y\right)}{y_{v^{\prime}(i)}-y_{v^{\prime}(i+1)}}
\end{aligned}
$$

which, together with the fact that $v(i)=v^{\prime}(i+1)$ and $v(i+1)=v^{\prime}(i)$, implies (3.2).

Let $\leq$ denote the (strong) Bruhat order on permutations of $S_{n}$. Recall that the Bruhat order is the closure of the following covering relation: For $u, v \in S_{n}$, we say that $v$ covers $u$ if there exists a transposition $t_{i j}$ such that $v=u t_{i j}$ and $\ell(v)=\ell(u)+1$. The following lemma is known, see [4, Corollary 2.4] and the references therein.

Lemma 3.3. We have $\mathfrak{G}_{u}\left(y_{v} ; y\right)=0$ whenever $u \not \leq v$ in the Bruhat order.

Proof. The idea in the proof of [11, (2.22)] for double Schubert polynomials applies to double Grothendieck polynomials, and we include a proof here for the reader's convenience. Use descending induction on $\ell(u)$. The initial case is $u=w_{0}$. Since $u \not \leq v$, we have $v \neq w_{0}$. It is easily checked from (2.1) that $\mathfrak{G}_{w_{0}}\left(y_{v} ; y\right)=0$.

We now consider the case $u \neq w_{0}$. Choose a position $i$ such that $u(i)<u(i+1)$. Note that $u<u s_{i}$. Since $u \not \leq v$, we must have $u s_{i} \not \leq v$. We further claim that $u s_{i} \not \leq v s_{i}$. This can be seen as follows. We have either $v s_{i}<v$ or $v<v s_{i}$ (depending on which of $\ell\left(v s_{i}\right)$ and $\ell(v)$ is larger). If $v s_{i}<v$, then it is clear that $u s_{i} \not \leq v s_{i}$ since otherwise there would hold $u \leq v$. It remains to verify the case $v<v s_{i}$. Suppose to the contrary that $u s_{i} \leq v s_{i}$. Then $u<v s_{i}$. Since $v s_{i}>v$ and $u s_{i}>u$, applying the Lifting Property (see [3, Proposition 2.2.7]) to $u^{-1}$ and $\left(v s_{i}\right)^{-1}$, we obtain that $u \leq v$, leading to a contradiction. Now, by the definition in (2.2) and by the induction hypothesis,

$$
\mathfrak{G}_{u}\left(y_{v} ; y\right)=\frac{y_{v(i)} \mathfrak{G}_{u s_{i}}\left(y_{v} ; y\right)-y_{v(i+1)} \mathfrak{G}_{u s_{i}}\left(y_{v s_{i}} ; y\right)}{y_{v(i)}-y_{v(i+1)}}=0,
$$

as desired.

Lemma 3.4. Let $u \in S_{n}$ and $u^{\prime}=u s_{i}$ for some $i$ such that $\ell\left(u s_{i}\right)<\ell(u)$. Then,

$$
\mathfrak{G}_{u}\left(y_{u} ; y\right)=\left(1-\frac{y_{u(i+1)}}{y_{u(i)}}\right) \mathfrak{G}_{u^{\prime}}\left(y_{u^{\prime}} ; y\right)
$$


Proof. Apply Lemma 3.1 to $v=u$ and $v^{\prime}=u^{\prime}$. The first addend on the right side vanishes due to Lemma 3.3 ,

Lemma 3.5 (Buch-Rimányi [4, Corollary 2.6]). For each $u \in S_{n}$, we have

$$
\mathfrak{G}_{u}\left(y_{u} ; y\right)=\prod_{\substack{i<j \\ u(i)>u(j)}}\left(1-\frac{y_{u(j)}}{y_{u(i)}}\right) .
$$

Proof. Make descending induction on $\ell(u)$. The induction base for $u=w_{0}$ is a restatement of (2.1). Assume that $u \neq w_{0}$. Then there exists some $1 \leq k<n$ such that $\ell\left(u s_{k}\right)>\ell(u)$. Let $u^{\prime}=u s_{k}$. It is easy to see that the set

$$
\left\{\left(u^{\prime}(i), u^{\prime}(j)\right) \mid i<j, u^{\prime}(i)>u^{\prime}(j)\right\}
$$

is the union of the two disjoint sets

$$
\{(u(i), u(j)) \mid i<j, u(i)>u(j)\} \cup\{(u(k), u(k+1))\} .
$$

The proof follows by induction together with Lemma 3.4.

Proof of Theorem 2.1. The proof is by induction on $\ell(v)$. Let us first consider the case $\ell(v)=0$, that is, $v$ is the identity permutation $e$. If $u=e$, then it follows from Lemma 3.5 (applied to $u=e$ ) that $\mathfrak{G}_{e}\left(y_{e} ; y\right)=1$. If $u \neq e$, then Lemma 3.3 forces that $\mathfrak{G}_{u}\left(y_{e} ; y\right)=0$. So (2.4) holds for $\ell(v)=0$.

Assume now that $\ell(v)>0$. Let $s_{r}$ be the last descent of $v$, that is, $r$ is the largest index such that $v(r)>v(r+1)$. Write $v=v^{\prime} s_{r}$. Clearly, the bottom row of $D(v)$ lies in row $r$ of $\Delta_{n}$. The leftmost square in the bottom row of $D(v)$, denoted $B_{0}$, has weight

$$
\mathrm{wt}\left(B_{0}\right)=1-\frac{y_{v(r+1)}}{y_{v(r)}}=1-\frac{y_{v^{\prime}(r)}}{y_{v^{\prime}(r+1)}} .
$$

Let $u=u^{\prime} s_{r}$. There are two cases.

Case 1. $s_{r}$ is a descent of $u$. By Lemma 3.1 and by induction hypothesis, we have

$$
\begin{aligned}
\mathfrak{G}_{u}\left(y_{v} ; y\right)= & \frac{y_{v^{\prime}(r)}}{y_{v^{\prime}(r+1)}} \mathfrak{G}_{u}\left(y_{v^{\prime}} ; y\right)+\left(1-\frac{y_{v^{\prime}(r)}}{y_{v^{\prime}(r+1)}}\right) \mathfrak{G}_{u^{\prime}}\left(y_{v^{\prime}} ; y\right) \\
= & \left(1-\operatorname{wt}\left(B_{0}\right)\right) \sum_{D \in \mathcal{H}\left(u, v^{\prime}\right)}(-1)^{|D|-\ell(u)} \mathrm{wt}(D)+\operatorname{wt}\left(B_{0}\right) \sum_{D \in \mathcal{H}\left(u^{\prime}, v^{\prime}\right)}(-1)^{|D|-\ell\left(u^{\prime}\right)} \operatorname{wt}(D) \\
= & \sum_{D \in \mathcal{H}\left(u, v^{\prime}\right)}(-1)^{|D|-\ell(u)} \mathrm{wt}(D)-\operatorname{wt}\left(B_{0}\right) \sum_{D \in \mathcal{H}\left(u, v^{\prime}\right)}(-1)^{|D|-\ell(u)} \mathrm{wt}(D) \\
& +\operatorname{wt}\left(B_{0}\right) \sum_{D \in \mathcal{H}\left(u^{\prime}, v^{\prime}\right)}(-1)^{|D|-\ell\left(u^{\prime}\right)} \operatorname{wt}(D) .
\end{aligned}
$$

To proceed, note that there is an obvious bijection $\phi$ between $D\left(v^{\prime}\right)$ and $D(v) \backslash\left\{B_{0}\right\}$. Since $s_{r}$ is the last descent of $v$, we have $c\left(v^{\prime}, r\right)=0, c\left(v^{\prime}, r+1\right)=c(v, r)-1$, and $c\left(v^{\prime}, i\right)=c(v, i)$ 
for $i \neq r, r+1$. Let $B \in D\left(v^{\prime}\right)$. If $B$ lies above row $r$, then set $\phi(B)=B$. Assume that $B$ lies in row $r+1$ and column $j$, then let $\phi(B)$ be the square of $D(v) \backslash\left\{B_{0}\right\}$ in row $r$ and column $j+1$. By construction, $B$ and $\phi(B)$ are labeled by the same simple transposition. Moreover, it is easy to see that $\phi$ preserves the weight and words, namely, $\operatorname{wt}(B)=\operatorname{wt}(\phi(B))$ and $\operatorname{word}(\phi(D))=\operatorname{word}(D)$ for all $D \subseteq D\left(v^{\prime}\right)$. Thus Hecke $(\phi(D))=\operatorname{Hecke}(D)$ for all $D \subseteq D\left(v^{\prime}\right)$.

We claim that $\mathcal{H}(u, v)$ is the disjoint union of the following sets:

$$
\begin{aligned}
& S_{1}=\left\{\phi(D): D \in \mathcal{H}\left(u, v^{\prime}\right)\right\}, \\
& S_{2}=\left\{\phi(D) \cup\left\{B_{0}\right\}: D \in \mathcal{H}\left(u, v^{\prime}\right)\right\}, \\
& S_{3}=\left\{\phi(D) \cup\left\{B_{0}\right\}: D \in \mathcal{H}\left(u^{\prime}, v^{\prime}\right)\right\} .
\end{aligned}
$$

This can be easily seen as follows. Keep in mind that $B_{0}$ is labeled by $s_{r}$. Let $D \in \mathcal{H}(u, v)$. If $B_{0} \notin D$, then $D \in S_{1}$. If $B_{0} \in D$, then word $(D)$ is obtained from word $\left(D \backslash\left\{B_{0}\right\}\right)$ by appending the letter $s_{r}$ at the end, and thus we have Hecke $(D)=\operatorname{Hecke}\left(D \backslash\left\{B_{0}\right\}\right) * s_{r}$, and therefore either $\operatorname{Hecke}\left(D \backslash\left\{B_{0}\right\}\right)=u$ or Hecke $\left(D \backslash\left\{B_{0}\right\}\right)=u^{\prime}$. Hence either $D \in S_{2}$ or $D \in S_{3}$. Conversely, any $D \in S_{1} \cup S_{2} \cup S_{3}$ belongs to $\mathcal{H}(u, v)$, since $u * s_{r}=u^{\prime} * s_{r}=u$. By the above claim and in view of (3.3), we obtain that

$$
\mathfrak{G}_{u}\left(y_{v} ; y\right)=\sum_{D \in S_{1} \cup S_{2} \cup S_{3}}(-1)^{|D|-\ell(u)} \operatorname{wt}(D)=\sum_{D \in \mathcal{H}(u, v)}(-1)^{|D|-\ell(u)} \operatorname{wt}(D) .
$$

Case 2. $s_{r}$ is not a descent of $u$. Let $D \in \mathcal{H}(u, v)$. We claim that $B_{0} \notin D$. Suppose otherwise that $B_{0} \in D$. Consider $D^{\prime}=D \backslash\left\{B_{0}\right\}$. If $s_{r}$ is a descent of $\operatorname{Hecke}\left(D^{\prime}\right)$, then $\operatorname{Hecke}(D)=$ $\operatorname{Hecke}\left(D^{\prime}\right)$, while if $s_{r}$ is not a descent of $\operatorname{Hecke}\left(D^{\prime}\right)$, then Hecke $(D)=\operatorname{Hecke}\left(D^{\prime}\right) s_{r}$. In both cases, $s_{r}$ is a descent of $u=\operatorname{Hecke}(D)$, leading to a contradiction. Therefore, we see that $\mathcal{H}(u, v)=\left\{\phi(D) \mid D \in \mathcal{H}\left(u, v^{\prime}\right)\right\}$. By Lemma 3.2 and by induction hypothesis,

$$
\mathfrak{G}_{u}\left(y_{v} ; y\right)=\mathfrak{G}_{u}\left(y_{v^{\prime}} ; y\right)=\sum_{D \in \mathcal{H}\left(u, v^{\prime}\right)}(-1)^{|D|-\ell(u)} \mathrm{wt}(D)=\sum_{D \in \mathcal{H}(u, v)}(-1)^{|D|-\ell(u)} \mathrm{wt}(D) .
$$

This completes the proof.

Acknowledgments. We wish to thank the referees for valuable suggestions that greatly improve the presentation of this note. This work was supported by the National Natural Science Foundation of China (Grant No. 11971250).

\section{References}

[1] N. Bergeron and S. Billey, RC-graphs and Schubert polynomials, Experiment. Math. 2 (4) (1993), 257-269.

[2] S. Billey, W. Jockusch and R.P. Stanley, Some combinatorial properties of Schubert polynomials, J. Algebraic Combin. 2 (1993), 345-374.

[3] A. Björner and F. Brenti, Combinatorics of Coxeter Groups, Grad. Texts in Math., Vol. 231, Springer, New York, 2005. 
[4] A. Buch and R. Rimányi, Specializations of Grothendieck polynomials, C. R. Acad. Sci. Paris, Ser. I 339 (2004), 1-4.

[5] S. Fomin and A.N. Kirillov, Grothendieck polynomials and the Yang-Baxter equation, Proc. Formal Power Series and Alg. Comb. (1994), 183-190.

[6] S. Fomin and A.N. Kirillov, The Yang-Baxter equation, symmetric functions, and Schubert polynomials, in: Proceedings of the 5th Conference on Formal Power Series and Algebraic Combinatorics (Florence, 1993), Discrete Math. 153 (1996), 123-143.

[7] S. Fomin and R.P. Stanley, Schubert polynomials and the NilCoxeter algebra, Adv. Math. 103 (1994), 196-207.

[8] J.E. Humphreys, Reflection Groups and Coxeter Groups, Cambridge Studies in Advanced Mathematics, No. 29, Cambridge Univ. Press, Cambridge, 1990.

[9] A. Knutson and E. Miller, Gröbner geometry of Schubert polynomials, Ann. Math. 161 (2005), 1245-1318.

[10] A. Knutson and E. Miller, Subword complexes in Coxeter groups, Adv. Math. 184 (2004), $161-176$.

[11] T. Lam, S. Lee and M. Shimozono, Back stable Schubert calculus, arXiv:1806.11233v1.

[12] A. Lascoux and M.-P. Schützenberger, Structure de Hopf de l'anneau de cohomologie et de l'anneau de Grothendieck dune variété de drapeaux, C. R. Acad. Sci. Paris 295 (1982), 629-633.

[13] C. Lenart, S. Robinson and F. Sottile, Grothendieck polynomials via permutation patterns and chains in the Bruhat order, Amer. J. Math. 128 (2006), 805-848.

[14] I.G. Macdonald, Notes on Schubert Polynomials, Laboratoire de combinatoire et d'informatique mathématique (LACIM), Université du Québec á Montréal, Montreal, 1991.

[15] A. Yong and A. Woo, A Gröbner basis for the Kazhdan-Lusztig ideals, Amer. J. Math. 134 (2012), 1089-1137. 